\title{
High entropy and sluggish diffusion "core" effects in senary FCC Al-Co-Cr-Fe-Ni-Mn alloys
}

\author{
Abhishek Mehta* and Yongho Sohn
}

Advanced Materials Processing and Analysis Center

Department of Materials Science and Engineering

University of Central Florida, Orlando, FL 32816, USA

*Corresponding author: abhi@knights.ucf.edu

\section{Supplementary information}

Table S1. Average effective interdiffusion coefficients, activation energy and pre-exponential factor for $\mathrm{Al}, \mathrm{Co}, \mathrm{Cr}, \mathrm{Fe}, \mathrm{Ni}$, and $\mathrm{Mn}$ in BCC $\mathrm{Al}_{48} \mathrm{Ni}_{52}$ and $\mathrm{FCC} \mathrm{Co}{ }_{20} \mathrm{Cr}_{20} \mathrm{Fe}_{20} \mathrm{Ni}_{20} \mathrm{Mn}_{20}$ terminal alloys determined from $\mathrm{Al}_{48} \mathrm{Ni}_{52} \mathrm{vs} \mathrm{Co}_{20} \mathrm{Cr}_{20} \mathrm{Fe}_{20} \mathrm{Ni}_{20} \mathrm{Mn}_{20}$ diffusion couples.

\begin{tabular}{|c|c|c|c|c|c|c|c|}
\hline $\begin{array}{c}\text { Terminal Alloy } \\
\text { in Diffusion } \\
\text { couple }\end{array}$ & $\begin{array}{c}\text { Temperat } \\
\text { ure }\left({ }^{\circ} \mathrm{C}\right)\end{array}$ & $\overline{\tilde{D}}_{A l}^{e f f}\left(\mathrm{~m}^{2} / \mathrm{s}\right)$ & $\overline{\tilde{D}}_{C r}^{e f f}\left(\mathrm{~m}^{2} / \mathrm{s}\right)$ & $\overline{\tilde{\tilde{D}}}_{\mathrm{Fe}}^{\text {eff }}\left(\mathrm{m}^{2} / \mathrm{s}\right)$ & $\overline{\tilde{D}}_{C o}^{e f f}\left(\mathrm{~m}^{2} / \mathrm{s}\right)$ & $\overline{\tilde{D}}_{N i}^{\text {eff }}\left(\mathrm{m}^{2} / \mathrm{s}\right)$ & $\overline{\tilde{D}}_{M n}^{e f f}\left(\mathrm{~m}^{2} / \mathrm{s}\right)$ \\
\hline \multirow[t]{4}{*}{$\mathbf{A l}_{48} \mathbf{N i}_{52}$} & 900 & $3.66(0.98) \times 10^{-17}$ & $3.50(0.99) \times 10^{-17}$ & $4.22(1.30) \times 10^{-17}$ & $3.23(0.92) \times 10^{-17}$ & $3.34(1.18) \times 10^{-17}$ & $3.51(1.04) \times 10^{-17}$ \\
\hline & 1100 & $2.93(0.02) \times 10^{-14}$ & $2.24(0.18) \times 10^{-14}$ & $2.82(0.12) \times 10^{-14}$ & $2.74(0.13) \times 10^{-14}$ & $2.65(0.09) \times 10^{-14}$ & $3.05(0.02) \times 10^{-14}$ \\
\hline & 1200 & $1.83(0.0) \times 10^{-13}$ & $1.46(0.08) \times 10^{-13}$ & $1.65(0.07) \times 10^{-13}$ & $1.82(0.01) \times 10^{-13}$ & $1.75(0.04) \times 10^{-13}$ & $2.05(0.03) \times 10^{-13}$ \\
\hline & $\mathrm{Q}(\mathrm{kJ} / \mathrm{mol})$ & 425.34 & 415.45 & 412.37 & 430.00 & 426.42 & 432.99 \\
\hline \multirow{5}{*}{$\begin{array}{c}\mathrm{Co}_{20} \mathrm{Cr}_{20} \mathrm{Fe}_{20} \mathrm{Ni}_{2} \\
{ }_{0} \mathrm{Mn}_{20}\end{array}$} & 1000 & $2.10(0.94) \times 10^{-15}$ & $1.63(0.28) \times 10^{-15}$ & $5.90(2.23) \times 10^{-16}$ & $4.16(1.92) \times 10^{-16}$ & $3.60(0.89) \times 10^{-16}$ & $1.78(0.59) \times 10^{-15}$ \\
\hline & 1100 & $4.08(0.16) \times 10^{-14}$ & $2.38(0.74) \times 10^{-14}$ & $3.36(1.18) \times 10^{-15}$ & $9.06(0.79) \times 10^{-15}$ & $4.27(2.20) \times 10^{-15}$ & $3.40(0.28) \times 10^{-14}$ \\
\hline & 1200 & $1.73(0.13) \times 10^{-13}$ & $1.90(0.80) \times 10^{-13}$ & $4.46(1.90) \times 10^{-14}$ & $4.17(0.87) \times 10^{-14}$ & $3.06(1.10) \times 10^{-14}$ & $1.90(0.16) \times 10^{-13}$ \\
\hline & $\mathrm{Q}(\mathrm{kJ} / \mathrm{mol})$ & 325.08 & 387.68 & 312.77 & 315.02 & 318.84 & 353.68 \\
\hline & $D_{0}\left(\mathrm{~m}^{2} / \mathrm{s}\right)$ & 0.0658 & 11.948 & 0.0041 & 0.0063 & 0.0057 & 0.7387 \\
\hline
\end{tabular}


Table S2. Comparison of average effective interdiffusion coefficients of $\mathrm{Al}, \mathrm{Co}, \mathrm{Cr}, \mathrm{Fe}, \mathrm{Ni}$, and $\mathrm{Mn}$ at $1000{ }^{\circ} \mathrm{C}$ in various $\mathrm{FCC} \mathrm{Co}-$ $\mathrm{Cr}-\mathrm{Fe}-\mathrm{Ni}$ [1], Co-Cr-Fe-Ni-Mn [2], Al-Co-Cr-Fe-Ni [3], and Al-Co-Cr-Fe-Ni-Mn [present work] alloys.

\begin{tabular}{|c|c|c|c|c|c|c|}
\hline \multirow{3}{*}{$\begin{array}{l}\text { Elem } \\
\text { ent } \\
i\end{array}$} & \multicolumn{2}{|c|}{$\begin{array}{c}\mathrm{Co}_{20} \mathrm{Cr}_{30} \mathrm{Fe}_{20} \mathrm{Ni}_{30} \text { vs. } \\
\mathrm{Co}_{30} \mathrm{Cr}_{20} \mathrm{Fe}_{30} \mathrm{Ni}_{20}\end{array}$} & \multicolumn{2}{|c|}{$\mathrm{Co}_{10} \mathrm{Cr}_{20} \mathrm{Fe}_{15} \mathrm{Mn}_{25} \mathrm{Ni}_{30}$ vs. $\mathrm{Co}_{30} \mathrm{Cr}_{20} \mathrm{Fe}_{25} \mathrm{Mn}_{15} \mathrm{Ni}_{10}$} & $\begin{array}{c}\mathrm{Al}_{48} \mathrm{Ni}_{52} \mathrm{vs} . \\
\mathrm{Co}_{25} \mathrm{Cr}_{25} \mathrm{Fe}_{25} \mathrm{Ni}_{25}\end{array}$ & $\begin{array}{c}\mathrm{Al}_{48} \mathrm{Ni}_{52} \text { vs. } \\
\mathrm{Co}_{20} \mathrm{Cr}_{20} \mathrm{Fe}_{20} \mathrm{Ni}_{20} \mathrm{Mn}_{20}\end{array}$ \\
\hline & $\begin{array}{l}\mathrm{Co}_{30} \mathrm{Cr}_{20} \mathrm{Fe}_{30} \mathrm{Ni} \\
{ }_{20} \text { to } \\
\mathrm{Co}_{25} \mathrm{Cr}_{25} \mathrm{Fe}_{25} \mathrm{Ni} \\
{ }_{25}\end{array}$ & $\begin{array}{c}\mathrm{Co}_{25} \mathrm{Cr}_{25} \mathrm{Fe}_{25} \\
\mathrm{Ni}_{25} \text { to } \\
\mathrm{Co}_{30} \mathrm{Cr}_{20} \mathrm{Fe}_{30} \\
\mathrm{Ni}_{20}\end{array}$ & $\begin{array}{c}\mathrm{Co}_{10.3} \mathrm{Cr}_{19.2} \mathrm{Fe}_{15.1} \mathrm{Mn}_{25} \\
{ }_{5} \mathrm{Ni}_{29.7} \text { to } \\
\mathrm{Co}_{20.1} \mathrm{Cr}_{21.6} \mathrm{Fe}_{19.6} \mathrm{Mn}_{20} \\
{ }_{.7} \mathrm{Ni}_{18.4}\end{array}$ & $\begin{array}{c}\mathrm{Co}_{20.1} \mathrm{Cr}_{21.6} \mathrm{Fe}_{19.6} \mathrm{Mn}_{20.7} \mathrm{~N} \\
\mathrm{i}_{18.4} \text { to } \\
\mathrm{Co}_{29.4} \mathrm{Cr}_{20.8} \mathrm{Fe}_{25.1} \mathrm{Mn}_{15.6} \mathrm{~N} \\
\mathrm{i}_{9.8}\end{array}$ & $\begin{array}{c}\mathrm{Al}_{5.5} \mathrm{Co}_{20.9} \mathrm{Cr}_{25.4} \mathrm{Fe}_{2} \\
{ }_{3.9} \mathrm{Ni}_{24.3} \text { to } \\
\mathrm{Al}_{0} \mathrm{Co}_{25} \mathrm{Cr}_{25} \mathrm{Fe}_{25} \mathrm{Ni} \\
{ }_{25}\end{array}$ & $\begin{array}{c}\mathrm{Al}_{5.5} \mathrm{Co}_{16.4} \mathrm{Cr}_{22.2} \mathrm{Fe}_{21.1} \mathrm{~N} \\
\mathrm{i}_{21.8} \mathrm{Mn}_{13.0} \text { to } \\
\mathrm{Al}_{0} \mathrm{Co}_{20} \mathrm{Cr}_{20} \mathrm{Fe}_{20} \mathrm{Ni}_{20} \mathrm{M} \\
\mathrm{n}_{20}\end{array}$ \\
\hline & $\overline{\tilde{D}}_{i, L}^{\text {eff }}\left(\mathrm{m}^{2} / \mathrm{s}\right)$ & $\overline{\tilde{D}}_{i, R}^{\text {eff }}\left(\mathrm{m}^{2} / \mathrm{s}\right)$ & $\overline{\tilde{D}}_{i, L}^{\text {eff }}\left(\mathrm{m}^{2} / \mathrm{s}\right)$ & $\overline{\tilde{D}}_{i, R}^{\text {eff }}\left(\mathrm{m}^{2} / \mathrm{s}\right)$ & $\overline{\tilde{D}}_{i}^{\text {eff }}\left(\mathrm{m}^{2} / \mathrm{s}\right)$ & $\overline{\tilde{D}}_{i}^{\text {eff }}\left(\mathrm{m}^{2} / \mathrm{s}\right)$ \\
\hline $\mathrm{Fe}$ & $3.4 \times 10^{-16}$ & $3.0 \times 10^{-16}$ & $4.8 \times 10^{-16}$ & $3.7 \times 10^{-16}$ & $1.4 \times 10^{-15}$ & $5.9 \times 10^{-16}$ \\
\hline $\mathrm{Co}$ & $1.3 \times 10^{-16}$ & $1.8 \times 10^{-16}$ & $2.5 \times 10^{-16}$ & $2.4 \times 10^{-16}$ & $9.1 \times 10^{-16}$ & $4.2 \times 10^{-16}$ \\
\hline $\mathrm{Ni}$ & $0.9 \times 10^{-16}$ & $0.5 \times 10^{-16}$ & $1.9 \times 10^{-16}$ & $1.9 \times 10^{-16}$ & $-2.2 \times 10^{-15}$ & $3.6 \times 10^{-16}$ \\
\hline Mn & - & - & $2.7 \times 10^{-16}$ & $2.9 \times 10^{-16}$ & - & $1.8 \times 10^{-15}$ \\
\hline $\mathrm{Al}$ & - & - & - & - & $9.3 \times 10^{-16}$ & $2.1 \times 10^{-15}$ \\
\hline
\end{tabular}


Table S3. Comparison of average effective interdiffusion coefficients of $\mathrm{Fe}, \mathrm{Cr}$, and $\mathrm{Ni}$ at $1100{ }^{\circ} \mathrm{C}$ in $\mathrm{Fe}-\mathrm{Cr}-\mathrm{Ni}$ [4] alloy with average effective interdiffusion coefficients of $\mathrm{Fe}, \mathrm{Cr}$, and $\mathrm{Ni}$ in $\mathrm{Al}-\mathrm{Co}-\mathrm{Cr}-\mathrm{Fe}-\mathrm{Ni}$ [3] and Al-Co-Cr-Fe-Ni-Mn [present work] alloys.

\begin{tabular}{|c|c|c|c|c|}
\hline \multirow{3}{*}{$\begin{array}{c}\text { Element } \\
i\end{array}$} & \multicolumn{2}{|c|}{$\mathrm{Cr}_{32.1} \mathrm{Fe}_{15.8} \mathrm{Ni}_{52.1}$ vs. $\mathrm{Cr}_{0} \mathrm{Fe}_{52} \mathrm{Ni}_{48}$} & $\mathrm{Al}_{48} \mathrm{Ni}_{52}$ vs. $\mathrm{Co}_{25} \mathrm{Cr}_{25} \mathrm{Fe}_{25} \mathrm{Ni}_{25}$ & $\mathrm{Al}_{48} \mathrm{Ni}_{52}$ vs. $\mathrm{Co}_{20} \mathrm{Cr}_{20} \mathrm{Fe}_{20} \mathrm{Ni}_{20} \mathrm{Mn}_{20}$ \\
\hline & $\begin{array}{l}\mathrm{Cr}_{32.1} \mathrm{Fe}_{15.8} \mathrm{Ni}_{52.1} \text { to } \\
\mathrm{Cr}_{16.1} \mathrm{Fe}_{33.5} \mathrm{Ni}_{50.4}\end{array}$ & $\begin{array}{rr}\mathrm{Cr}_{16.1} \mathrm{Fe}_{33.5} \mathrm{Ni}_{50.4} \text { to } \\
\mathrm{Cr}_{0} \mathrm{Fe}_{52} \mathrm{Ni}_{48}\end{array}$ & $\begin{array}{c}\mathrm{Al}_{8.6} \mathrm{Co}_{17.2} \mathrm{Cr}_{25.6} \mathrm{Fe}_{20.2} \mathrm{Ni}_{28.3} \text { to } \\
\mathrm{Al}_{0} \mathrm{Co}_{25} \mathrm{Cr}_{25} \mathrm{Fe}_{25} \mathrm{Ni}_{25}\end{array}$ & $\begin{array}{c}\mathrm{Al}_{7.3} \mathrm{Co}_{14.8} \mathrm{Cr}_{21.5} \mathrm{Fe}_{18.8} \mathrm{Ni}_{25.7} \mathrm{Mn}_{11.9} \text { to } \\
\mathrm{Al}_{0} \mathrm{Co}_{20} \mathrm{Cr}_{20} \mathrm{Fe}_{20} \mathrm{Ni}_{20} \mathrm{Mn}_{20}\end{array}$ \\
\hline & $\overline{\tilde{D}}_{i, L}^{\text {eff }}\left(\mathrm{m}^{2} / \mathrm{s}\right)$ & $\overline{\tilde{D}}_{i, R}^{\text {eff }}\left(\mathrm{m}^{2} / \mathrm{s}\right)$ & $\overline{\tilde{D}}_{i}^{e f f}\left(\mathrm{~m}^{2} / \mathrm{s}\right)$ & $\overline{\tilde{D}}_{i}^{e f f}\left(\mathrm{~m}^{2} / \mathrm{s}\right)$ \\
\hline $\mathrm{Cr}$ & $6.5 \times 10^{-15}$ & $6.2 \times 10^{-15}$ & $-2.7 \times 10^{-14}$ & $2.38 \times 10^{-14}$ \\
\hline $\mathrm{Fe}$ & $5.4 \times 10^{-15}$ & $5.5 \times 10^{-15}$ & $1.1 \times 10^{-14}$ & $3.36 \times 10^{-15}$ \\
\hline $\mathrm{Co}$ & - & - & $1.5 \times 10^{-14}$ & $9.06 \times 10^{-15}$ \\
\hline $\mathrm{Ni}$ & $\begin{array}{c}2.2 \times 10^{-16} \\
\text { (Uphill diffusion) }\end{array}$ & $1.7 \times 10^{-15}$ & $-1.2 \times 10^{-14}$ & $4.27 \times 10^{-15}$ \\
\hline $\mathrm{Al}$ & - & - & $2.1 \times 10^{-14}$ & $4.08 \times 10^{-14}$ \\
\hline $\mathrm{Mn}$ & - & - & - & $3.40 \times 10^{-14}$ \\
\hline
\end{tabular}

\section{REFERENCES}

1. Kulkarni, K.; Chauhan, G. P. S. Investigations of quaternary interdiffusion in a constituent system of high entropy alloys. AIP Advances 2015, 5 (9), 097162.

2. Verma, V.; Tripathi, A.; Kulkarni, K. N. On interdiffusion in FeNiCoCrMn high entropy alloy. Journal of Phase Equilibria and Diffusion 2017, 38 (4), 445-456.

3. Mehta, A.; Sohn, Y. H. Interdiffusion, Solubility Limit, and Role of Entropy in FCC Al-Co-Cr-Fe-Ni Alloys. Metallurgical and Materials Transactions A 2020, 51 (6), 3142-3153.

4. Duh, J. G.; Dayananda, M. A. Interdiffusion in Fe-Ni-Cr Alloys at $1100^{\circ}$ C. Diffusion and Defect Data 1985, 39, 1-50. 